\title{
Optimizing community services for an improved continuum of HIV
} care

Project SOAR

Follow this and additional works at: https://knowledgecommons.popcouncil.org/departments_sbsr-hiv

Part of the Demography, Population, and Ecology Commons, Family, Life Course, and Society Commons, International Public Health Commons, and the Medicine and Health Commons How does access to this work benefit you? Let us know!

\section{Recommended Citation}

Project SOAR. 2017. "Optimizing community services for an improved continuum of HIV care," Activity brief. Washington, DC: Population Council. 


\section{Optimizing Community Services for an Improved Continuum of HIV Care in South Africa}

Extending care beyond the traditional clinic approach is essential to reaching UNAIDS' ambitious HIV treatment and viral suppression goals. Such care is often referred to as community care and may include community support and adherence clubs, nutrition and economic support, and delivery of medication refills and basic clinical evaluations. These and other strategies are currently being introduced within communities leading to a varied and heterogeneous array of services in many communities. Yet there has been little systematic planning in selecting and delivering community care services that best respond to the community's HIV treatment needs.

Project SOAR, together with Aurum Institute, is conducting research in Ekurhuleni District and Bojanala District to improve the availability of effective care continuum services. Ekurhuleni has the second-highest district level HIV prevalence in South Africa at 14.3 percent ${ }^{1}$ while Bojanala has among the highest antenatal HIV prevalence (33.9 percent) in the country. ${ }^{2}$ The two districts have a combined population of

Research Partners: Aurum Institute

Location: Ekurhuleni and Bojanala Districts

Study Duration: 2015-2018

For more information, contact

Christopher Hoffmann, choffmann@jhmi.edu or Tonderai Mabuto,tmabuto@auruminstitute. org.

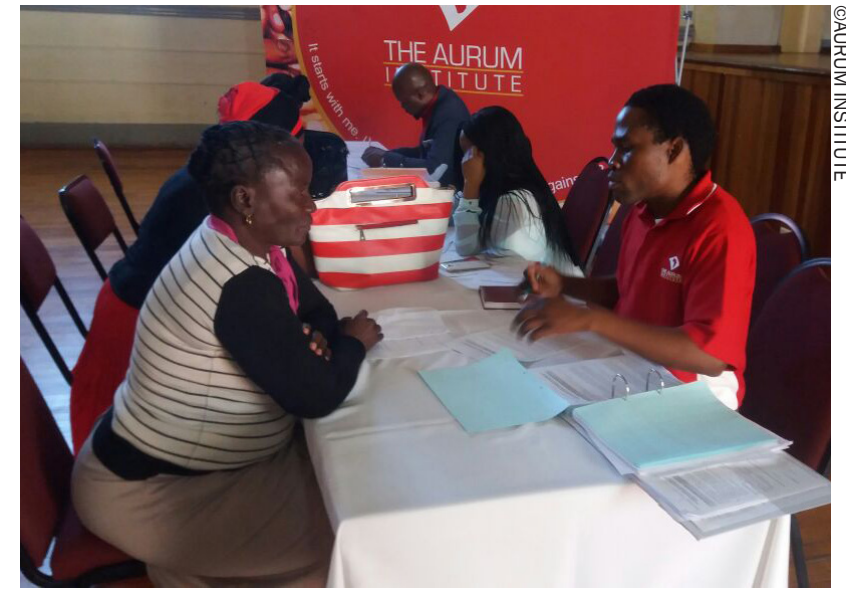

This study will provide valuable new knowledge about gaps and effective approaches to planning and delivering services that best achieve HIV treatment goals in South Africa.

3.6 million inhabitants and approximately 350 public clinics and hospitals. Through improved information on community HIV care services and their relationship to care continuum outcomes, the study will provide valuable new knowledge about service gaps and approaches to planning and delivering services that best achieve HIV treatment goals.

\section{Our Research}

We will conduct structured interviews to identify the types and number of services available and the human and material resources to deliver them in each clinic's catchment area. These interviews will be with clinic managers or district/ sub-district HIV coordinators, nongovernmental organizations involved in service delivery or health system strengthening, and ward counselors and/ or ward-based community health workers (CHWs). The data will provide a description of available services and their reach. 


\section{What are the study objectives?}

- Determine what community and clinic services are available in the catchment areas of 100 community clinics each in Ekurhuleni and Bojanala Districts.

- Identify associations between community and clinic-based services and care continuum outcomes.

- Develop a worksheet to easily assess availability of services associated with improved care continuum outcomes.

- Pilot test use of the worksheet and utilization of the worksheet results to bring the identified services to the community.

We will obtain HIV continuum of care outcome data from the clinic-level electronic data system. We will specifically focus on the outcomes of 12 and 24 month retention in care among ART patients and 12 and 24 month levels of viral suppression (HIV RNA $<400 \mathrm{c} / \mathrm{mL}$ ). We will then assess the relationships between the number, types, and other characteristics of the community services and HIV care continuum outcomes

The research team will adapt an existing worksheet to rapidly identify: 1 ) available community services, 2) unmet needs of people living with HIV (PLHIV), and 3) potential community-based services to meet those needs. To inform its adaptation we will conduct focus group discussions with community PLHIV groups, CHWs, local political and traditional leadership, clinic staff, and others. Once the worksheet is ready we will train 2-5 CHWs in each of 10-12 catchment areas in the use of the worksheets.
We will use structured interviews with $\mathrm{CHWs}$ and clinic health workers to determine how well the worksheet used by the CHWs identified gaps and needed services, and the acceptability of the worksheet process. After six months we will examine whether the recommended services are being implemented and the views of CHWs, PLHIV, and clinic staff on the implementation process and subsequent changes.

\section{Research Utilization}

The key audiences of our findings are national, provincial, and Ekurhuleni and Bojanala district/ subdistrict departments of health and health system implementing partners. These audiences include those closest to the ground-the $\mathrm{CHWs}$ in the study catchment areas-to the South African National Department of Health. Other specific audiences include the Ekurhuleni and Bojanala HIV/AIDS and Sexually Transmitted Diseases coordination bodies, the Ekurhuleni and Bojanala Health Services Committees, the Ekurhuleni and Bojanala Research Committees, the Gauteng Provincial Ministry of Health, and representatives of civil society. We will reach these audiences and foster utilization by engaging them throughout the study period through 1) a study advisory committee; 2) interactive presentations; and 3) email updates. 1Shisana, O. et al. 2012. South African National HIV
Prevalence, Incidence and Behaviour Survey, 2012. Cape
Town: HSRC Press.

${ }^{2}$ National Department of Health. The 2011 National Antenatal Sentinel HIV and Syphilis Prevalence Survey in South Africa. Pretoria: National Department of Health. agreement funded by the President's Emergency Plan for AIDS Relief and the U. S. Agency for International Development (Agreement No. AIDOAA-A-14-00060). SOAR is able to accept funding from all USAID accounts.
Project SOAR/Population Council

4301 Connecticut Avenue, NW, Suite 280

Washington, DC 20008

Tel: +1 2022379400

e-mail: ProjectSOAR@popcouncil.org

popcouncil.org/ProjectSOAR

(c)Population Council, May 2017 